\title{
A Common Measurement Method for Throughput Experiment of IEEE 802.11ac and IEEE 802.11n Wi-Fi under Variable Condition
}

\author{
Chang Choon Chew ${ }^{1}$, Nobuo Funabiki ${ }^{2}$ and Tutut Herawan ${ }^{3,4,5}$ \\ ${ }^{1}$ Embedded Computing Systems Group, Department of Computer Engineering, \\ Faculty of Electrical and Electronic Engineering, Universiti Tun Hussein Onn \\ Malaysia \\ ${ }^{2}$ Graduate School of Natural Science and Technology, Okayama University \\ ${ }^{3}$ Universitas Negeri Yogyakarta, Indonesia \\ ${ }^{4}$ Universitas Teknologi Yogyakarta, Indonesia \\ ${ }^{5}$ Politeknik Negeri Malang, Indonesia \\ chewcc@uthm.edu.my
}

\begin{abstract}
The IEEE 802.11ac wireless communication proto-cols have been introduced for highspeed wireless networks. Then, commercial devices implementing this protocol have been available from most vendors. In this paper, we provide various Transmission Control Protocol (TCP) throughput measurement results. It uses devices from three vendors implementing this protocol under a set of TCP parameters in various conditions including comparison between indoor and outdoor, comparison between one-hop and two-hop, effects of walls, effect of 11 ac repeater, big room with different AP locations, and effects of link distance. As a comparison, we also conduct the test in 11 n as well. Our measurement results show that TCP throughputs are greatly affected by vendors, communication, and physical conditions.
\end{abstract}

Keywords: Measurement; IEEE 802.11; Transmission Control Protocol; Wi-Fi; Variable Condition

\section{Introduction}

Recently, the 5GHz IEEE 802.11ac protocol has become popular as a high-speed wireless communication protocol and it's replacing the $2.4 \mathrm{GHz}$ IEEE $802.11 \mathrm{n}$ protocol in most communication devices with backward compatible support. It enhances the data transfer rate and wider signal coverage area by adopting the multiple-input-multiple-output (MIMO) channel, the multi-user MIMO, the frame aggregation, the channel bonding, and other technologies [1]. Inexpensive commercial products implementing this protocol have become available, including APs, USB Wi-Fi adapters and Network Interface Cards (NIC) for personal computers. Devices from some vendors allow up to two hop communications between them.

However, few throughput measurement results using IEEE 802.11ac devices from different vendors have been reported within our surveys [2][3][4], although they are important to deploy wireless local area networks (WLANs) in fields using them properly. Particularly, TCP throughputs should be observed because common network services such as World-Wide Webs and emails are using TCP, it is the key to Internet. It could possible serve as a reference to user for the allocation of TCP at the most ideal position in order to retrieve optimum Internet speed. Therefore, in this paper, we provide TCP throughput

Received (February 26, 2018), Review Result (May 23, 2018), Accepted (May 30, 2018) 
measurement results in various conditions in order to measure the performance of IEEE 802.11ac.

In our experiments, we employed IEEE 802.11ac devices from three vendors available in Japan, namely Buffalo, I-O Data, and NEC. The experiments took part both in outdoors and indoors. For outdoor experiments, the devices from the three vendors are used to measure throughput according to different link distances. For indoor experiments, they are used to measure throughput changes under different TCP parameters, different number of APs, existences of walls as obstacles along the link path as well as different AP positions in a big room. The devices from Buffalo and NEC are also used to measure throughput changes between one-hop and two-hop communications. Furthermore, throughput comparisons are also performed between devices using IEEE 802.11ac and devices using IEEE 802.11n.

The rest of this paper is organized as follows: Section 2 present actions required before conducting the experiment. Section 3 shows methods used to run the measurement test. Section 4 shows results. Section 5 concludes this paper with some future studies.

\section{Experiment Setup}

In this section, we explain the experiment setups for TCP throughput measurements using $11 \mathrm{ac}$ and $11 \mathrm{n}$ devices. There are few items to be taken into consideration as shown below before conducting the throughput measurement.
a. Configuration of Access Points (APs).
b. Usage of 11ac USB Wi-Fi Adapter
c. Experiment Sites.
d. Iperf Throughput Test Software.

\subsection{Configuration of Access Points (APs)}

For 11n, we adopted AP from Buffalo WZR-HP-G302H [5]. While for 11ac, we adopted APs from Buffalo WZR-1750DHP [6], I-O Data Air Port WN-AC1600DGR [7], and NEC Aterm WG1800HP [8][9]. In order to obtain the best throughput performance for these commercial APs, the latest firmware is downloaded. By referring to the AP's user manual for Buffalo WZR-HP-G302H[5], Buffalo WZR-1750DHP[6], IO Data WNAC1600DGR[7], and NEC Aterm WG1800HP[8][9] as following is performed.

a. Define common Local Area Network (LAN)-side IP address for every AP's configuration login page.

b. Define common wireless LAN's SSID, encryption type and encryption key for all experiment.

c. Define wireless LAN type, channel and bandwidth for different types of experiment.

d. Disable SSID broadcasting during experiment to avoid unnecessary connection and interference.

e. Update the AP's firmware through AP's login page at Internet web browser.

\subsection{Allocation of 11ac USB Wi-Fi Adapter}

In the throughput experiment, build-in 11n Wi-Fi device in the notebook PC enable user to directly communicate to an AP. Then, USB Wi-Fi devices for 11ac, namely Buffalo WIU2-866D[10], I-O Data WN-AC867U[11], and NEC Aterm WL900U[12], where devices from the same vendor were used for any experiment is connected through a USB port. Certain notebook PC will have their USB port located nearby the ventilation hole or heat exhaust. Waste heat generated from CPU and GPU's of notebook will be exhausted away, towards these ventilation holes and heat up these areas [13]. Take note that if we plugs in the Buffalo 11ac USB Wi-Fi adapter at the USB port near these area, the USB device itself will become very hot and it might exceed its standard operating environment's temperature 
of $0-40^{\circ} \mathrm{C}\left(32-104^{\circ} \mathrm{F}\right)[14]$. In order to avoid any operating error, we selected those USB port without any nearby ventilation hole.

\subsection{Experiment Sites}

Several experiment sites within Okayama University campus were selected to conduct our test. For the indoor part, we choose a few rooms and a corridor area near our laboratory. We also conduct the measurement at a big lecture hall. As for the outdoor part, we choose the open space road area nearby with our laboratory building.

\subsubsection{Indoor Experiment Sites}

For indoor experiment sites, we selected rooms and a corridor in our building (Engineering Building 2) in Figure 1 and in Figure 2. Variety kinds of test were conducted in the room and in between rooms in order to simulate the actual possible usage of Wi-Fi in room(s). The tests near the corridor area also simulate possible usage of Wi-Fi while user are standing around that area. To measure throughput drops by walls in indoor sites, we selected three types of setups. In the first setup, we measured throughput between the distances of three rooms partitioned by concrete walls in Figure 3. Then, we measured effects of a repeater in the middle room in Figure 4.

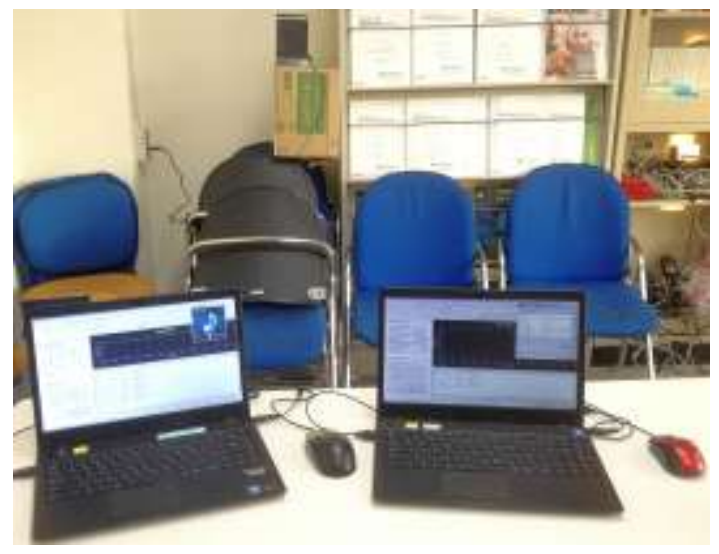

Figure 1. Clients for Indoor Experiments in Room

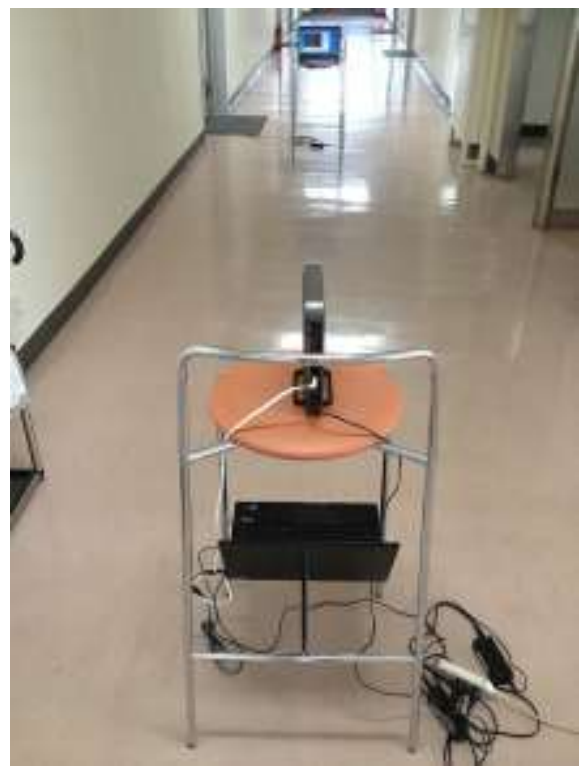

Figure 2. Server and Client for Indoor Experiments in Corridor 


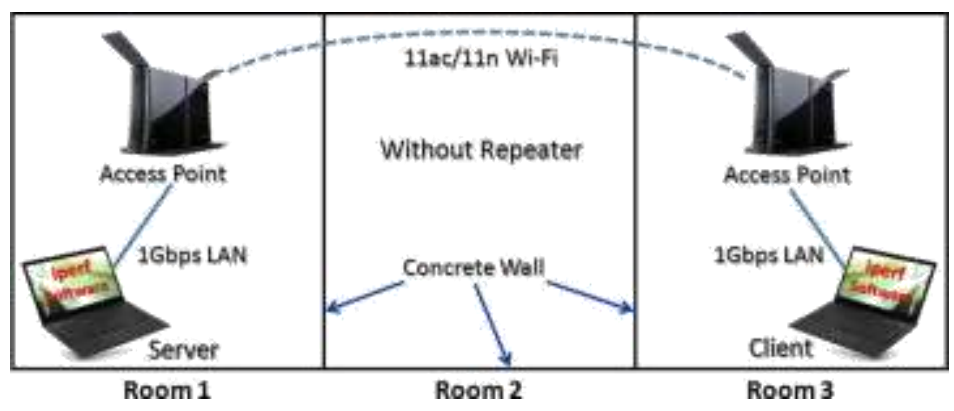

Figure 3. Indoor Experiment Setup in Three Rooms without Repeater Effects

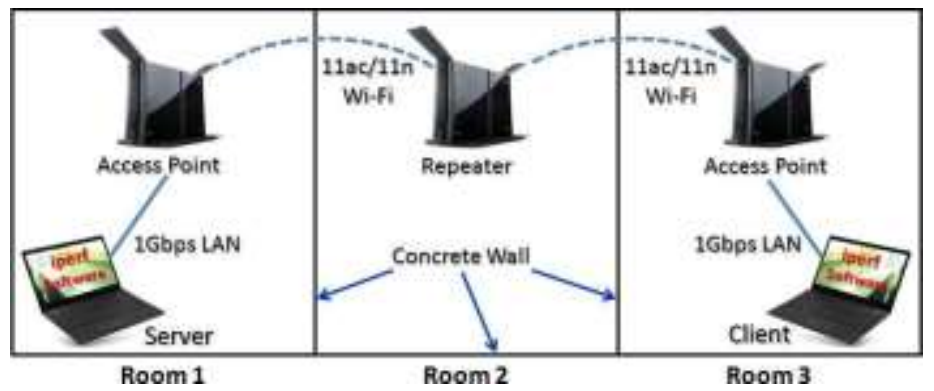

Figure 4. Indoor Experiment Setup in Three Rooms with Repeater Effects

In the second setup, we measured throughput between the distances of two rooms partitioned by one concrete wall. Here, we measured throughputs in both one-hop and twohop communications using the combination of $11 \mathrm{n}$ and 11 ac devices to evaluate the use of an 11 ac repeater for $11 \mathrm{n}$ clients as shown in Figure 5 and Figure 6. In the third setup, we measured throughput between the distances of three rooms partitioned by concrete walls. Here, we measured throughputs at fixed distance of $8 \mathrm{~m}$ between both host-AP configuration in Figure 7 and AP-AP configuration in Figure 8. We did the measurement using 11n and $11 \mathrm{ac}$ devices from three vendors to evaluate the effects of wall obstacles towards Wi-Fi signal. We also repeat the same test under one wall and no wall condition as shown in Figure 9, Figure 10, Figure 11, and Figure 12.

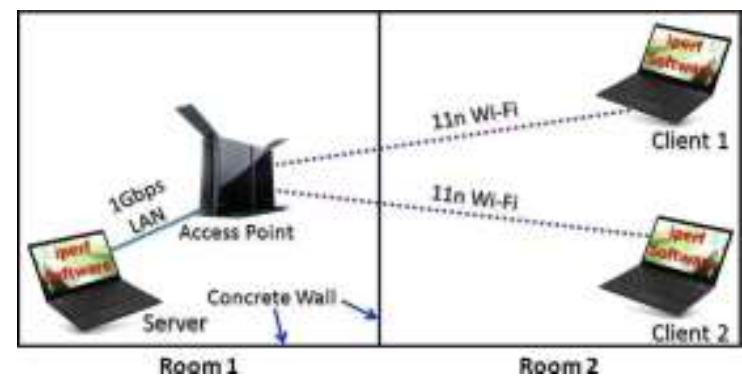

Figure 5. Indoor Experiment Setup without 11ac Repeater in Two Rooms

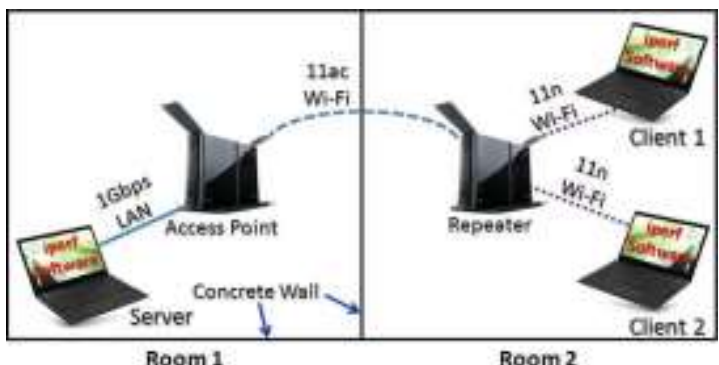

Figure 6. Indoor Experiment Setup with 11ac Repeater in Two Rooms 


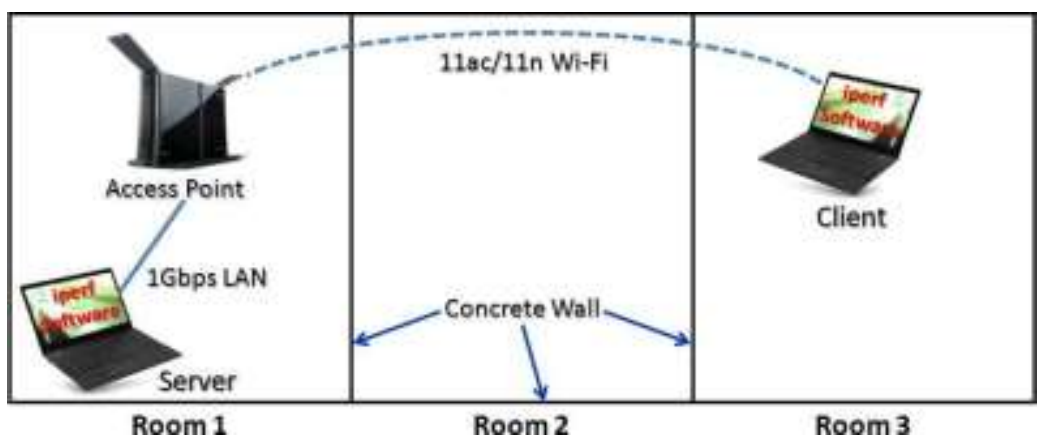

Figure 7. Host to AP Two Wall Test

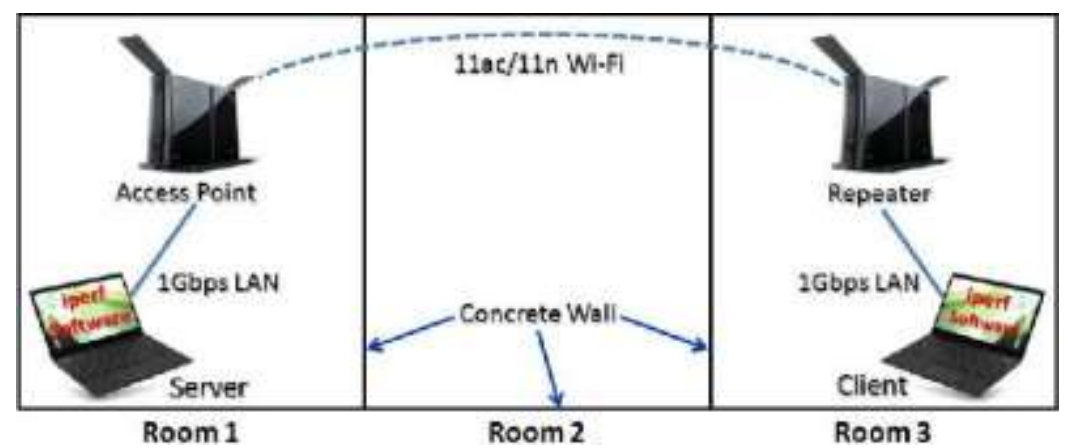

Figure 8. AP to AP Two Wall Test

\subsubsection{Lecture Hall Experiment Site}

Besides the throughput measurement within rooms, we had selected a big lecture hall. In order to cover the whole area of this room, nine hosts are placed regularly to simulate possible positions of users.

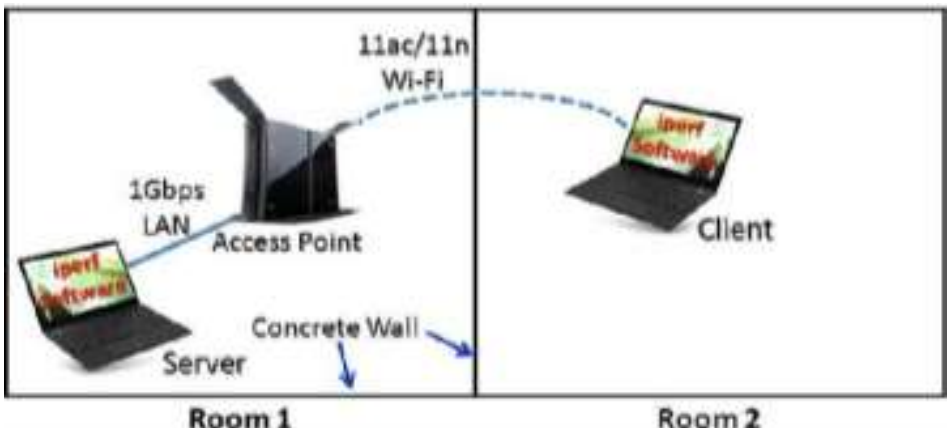

Figure 9. Host to AP One Wall Test

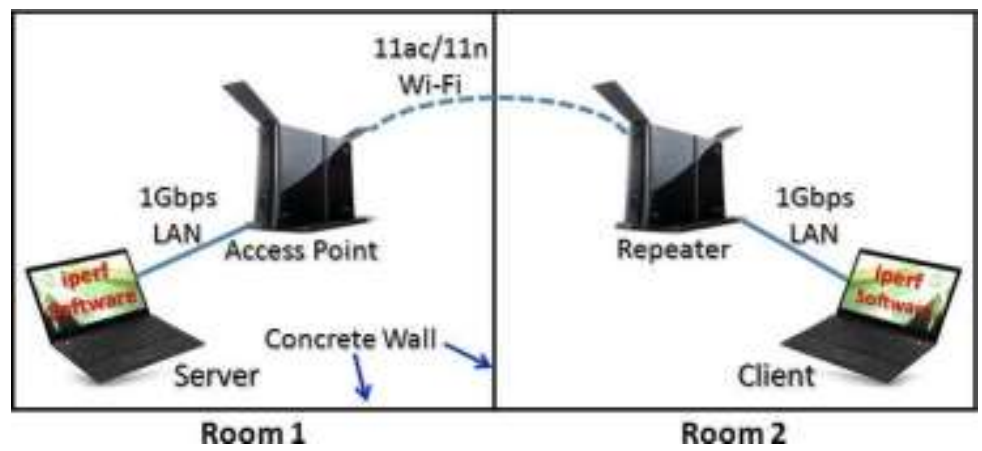

Figure 10. AP to AP One Wall Test 


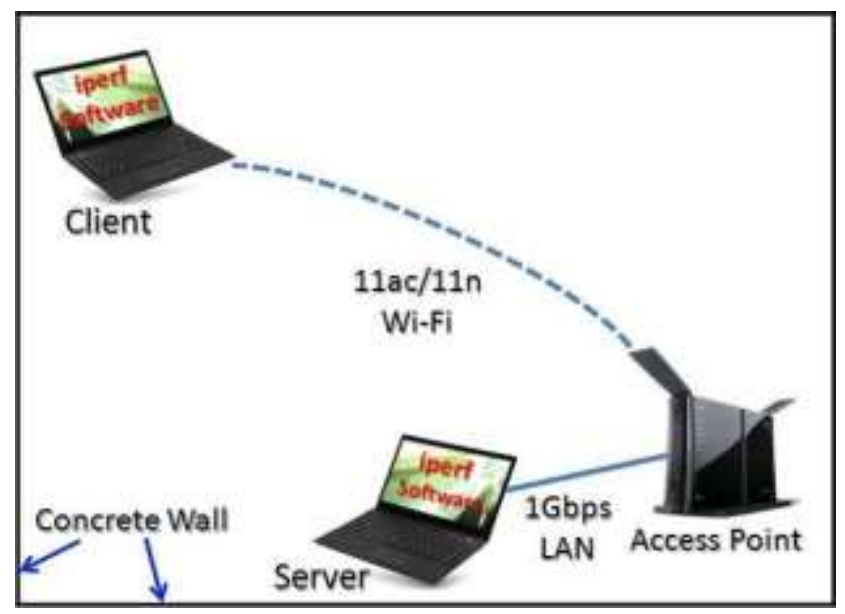

Figure 11. Host to AP No Wall Test

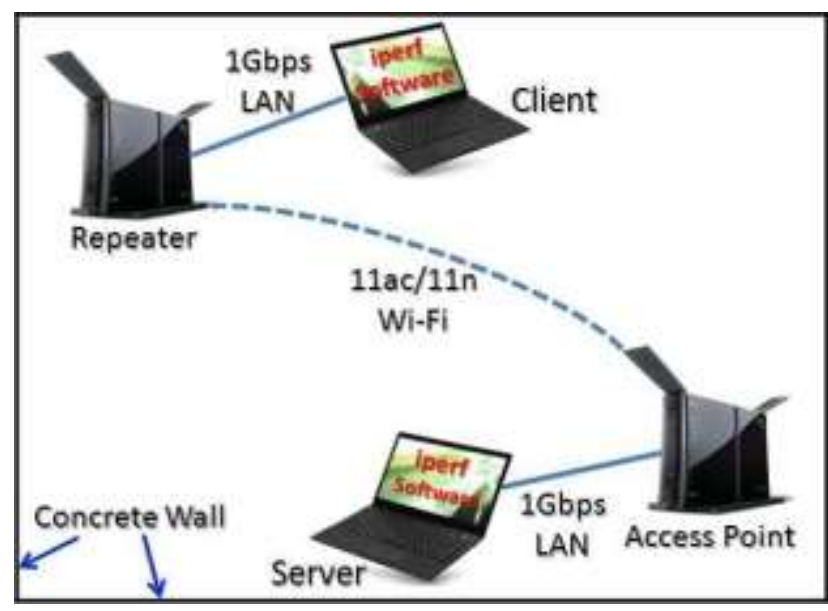

Figure 12. AP to AP No Wall Test

We had divided the experiment into two types of cases, namely idea case and practical case. Both cases are tested with multiple APs simulated as gateways. For idea case, in order to gain the highest throughput for one-AP case, we placed this AP in the middle of room as shown in Figure 13. For two-AP case, we divided the hosts into two groups and placed the AP in the middle of each group as shown in Figure 14. In practical case or real situations, any AP must be placed nearby from the Internet LAN cable port, and should not be an obstacle for the big lecture room user. Thus, we placed the one to three APs at the corner of the room at the Internet port as shown in Figure 15. The hosts are associated with one AP among them such that the number of hosts is nearly equal among the APs.

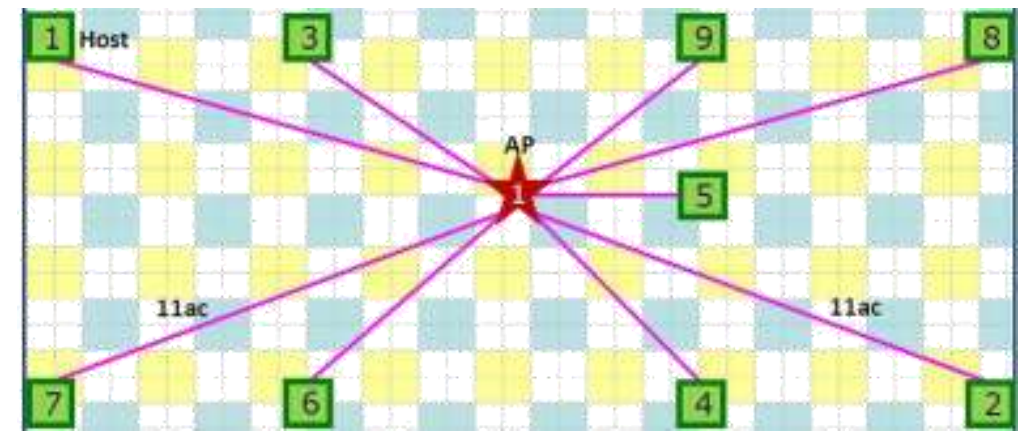

Figure 13. Throughputs with One AP for Ideal AP Location 


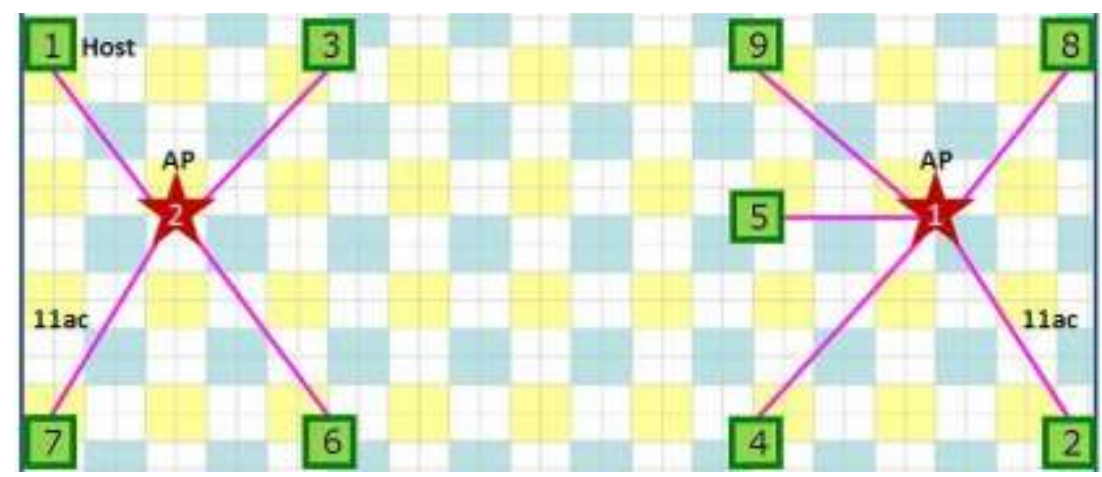

Figure 14. Two AP Topology for Ideal AP Location

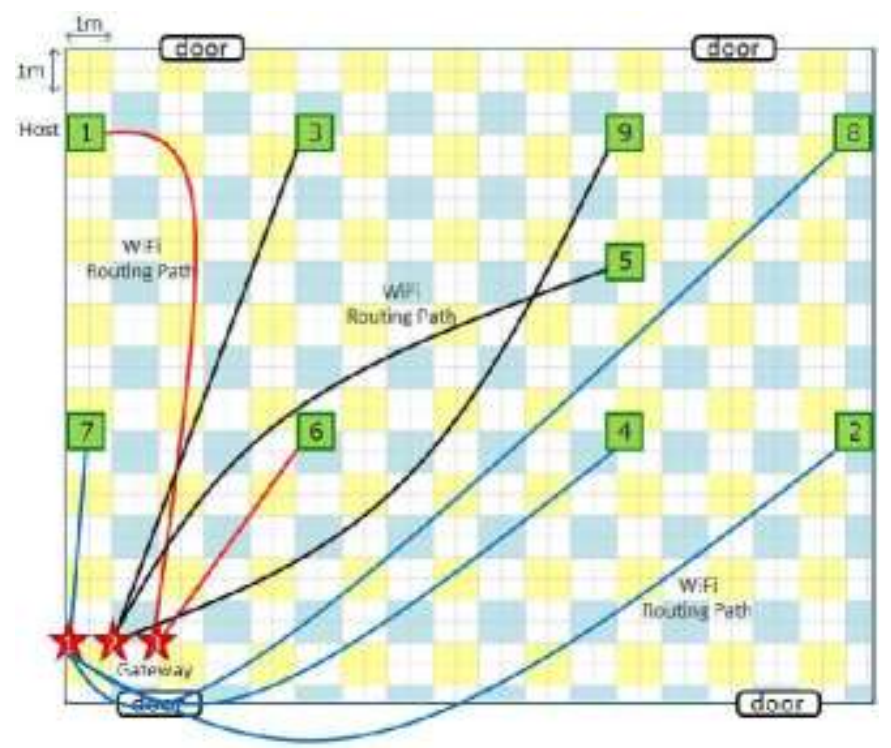

Figure 15. Three APs Topology for Practical AP Location

\subsection{Outdoor Experiment Site}

For the outdoor experiment site, we selected the open space in the campus near our building in Figure 16. We conducted the comparison test between 11 ac using Buffalo WZRG1750DHP APs and 11n with Buffalo WZR-G302H APs as shown in Figure 17 and Figure 18. We also did the comparison test using three $11 \mathrm{ac}$ vendors with Buffalo WZRG1750DHP AP, I-O Data AP, and NEC AP as well as their respective USB Wi-Fi adapter.
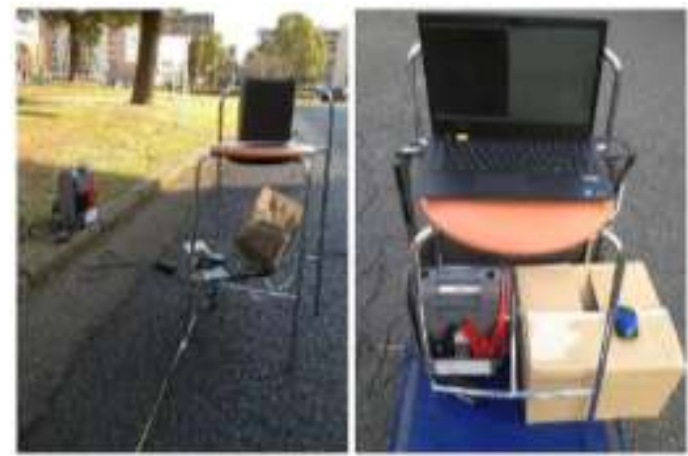

Figure 16. Server and Client for Outdoor Experiments in Campus 


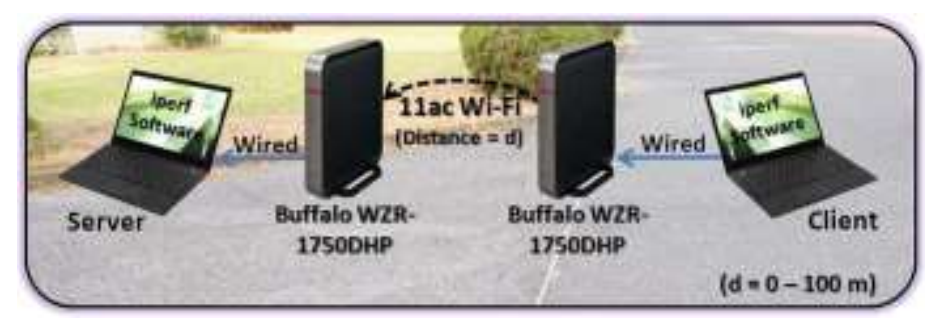

Figure 17. 11ac Outdoor Test

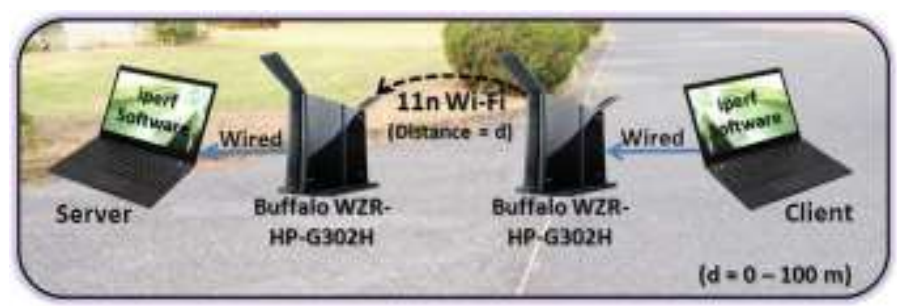

Figure 18. 11n Outdoor Test

\subsection{Iperf Throughput Test Software}

In our experiments, we used iperf v2.0.5 as a soft-ware tool to measure the TCP throughput. Iperf can accurately provide the detailed communication performance by setting the parameters in the software properly [18]. The default command for the server is iperf $-s$, and that for the client is iperf -c 192.168.2.102 -t 50 -i 10. During the throughput test, we had a total of nine hosts (refer as clients in iperf) using 11ac or 11n Wi-Fi connects to the AP (refer as a server in iperf). In order to run the whole test in a more efficient and accurate way, both server and hosts have been configured with batch files. The server will be executed with current date and time shown in two lines on the screen using the command "date $/ t$ and time $/ t$ ". Next, the iperf server command "iperf $-s-w 477 K-l 8 K$ " will be executed with TCP optimum window size of $477 \mathrm{~KB}$ and default buffer length of $8 \mathrm{~KB}$. With the maximum network bandwidth of $1300 \mathrm{Mbps}$ for $11 \mathrm{ac}$ and network delay of $3 \mathrm{~ms}$, this optimum window size can be calculated with Equation (1) using "SG Bandwidth*Delay Product Calculator" from Speed Guide, Inc. [19].

$$
\text { Windows Size }=\text { Network Bandwidth } \times \text { Delay }
$$

On the client side, two batch files are required during the whole throughput test.The first batch file "54.bat" will run the iperf client command "iperf -c 192.168.11.54 -t 50 -i 10 -w $477 K-l 8 K$ " for a duration of 50 seconds "- $t 50$ " and display the throughput info for every 10 seconds " $-i 10$ ". Client side also having the same TCP optimum window size of $477 \mathrm{~KB}$ and buffer length of $8 \mathrm{~KB}$ with using " $-w 477 \mathrm{~K}-l 8 K$ ". The "echo Start of 9 Hosts Test \& date $/ t \&$ time $/ t$ " and "echo End of 9 Hosts Test \& time / $t$ " commands will inform the user about the starting and ending of throughput test. As for the second batch file "51-54T7.bat", after 30 seconds of delay using "timeout 30 " command, it will execute the batch file "54.bat" again and save the whole process into a text file named "51-54-T7 140530.txt" with the command "54.bat >> 51-54-T7 140530.txt". As a result, all test results for every host will be automatically saved in a text file and this will ease the work of data sorting later.

\section{Experimental Setup}

This section will explain about how to optimize the network during every test and how to handle interference during measurement. 


\subsection{Network Optimization in Windows 7}

Take note that in order to optimize all network adapters (both build-in or external type), the configuration of network for all networking hardware in the notebook PC can be optimize with the SG TCP Optimizer version 3.0.8 from Speed Guide, Inc.[16]. This network optimizing process will ensure that all network adapter's maximum transmission unit (MTU) values are set to 1500 bytes [17] instead of default value at 0 bytes in Microsoft Windows 7.

\subsection{Wireless Networks Interference}

During the throughput measurement experiments, especially at indoor site, we observed lots of similar Wi-Fi signal with our devices (AP). Some of them might be in the same frequency $(2.4 \mathrm{GHz}$ or $5 \mathrm{GHz})$, some of them in the worst case, will be in the same Wi-Fi channel with our AP. In order to reduce the effects of these interferences towards our measurement, several steps are being taken into consideration. Depending on our experiment type, whether in $2.4 \mathrm{GHz}$ or $5 \mathrm{GHz}$ frequency, we will scan for the similar available Wi-Fi signal at the experiment site using both notebook PC and smart phone. In the notebook PC, we used Wi-Fi Channel Scanner software to detect such wireless networks. It is a real-time signal scanner for the current Wi-Fi hotspot that provides related network names (SSID), channels, signal quality, and other information [20]. While in the Android based version 4.2.2 operating system smart phone, we used WiFi Analyzer from [21] to check currently available channel for our AP(s). This software will show both $2.4 \mathrm{GHz}$ and $5 \mathrm{GHz}$ as in Figure 19. This application provides better portability as it allows the user to move around the test site to check the whole area for all available Wi-Fi signal.
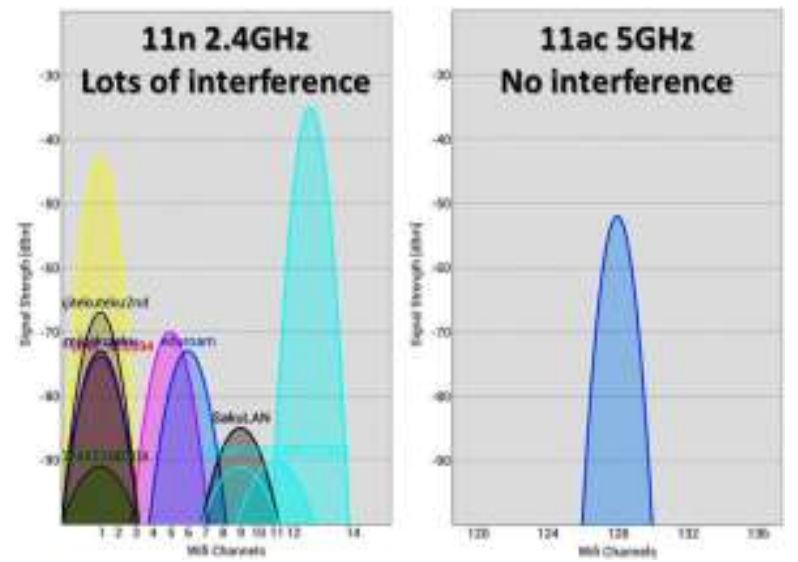

Figure 19. Observed 2.4GHz and 5GHz Wi-Fi Networks by Wifi Analyzer

Combining the channel scanning result from both PC and smart phone, we selected the least interfered Wi-Fi channel at the test site. If no suitable channel available during the measurement experiment, we will run test during off peak hour, such as mid night, weekend or holiday time, as these hours has the least number of user using Wi-Fi, resulting in less interference much accurate result towards our measurement.

\subsection{Thermal Fade Interference}

Thermal fade effect is a type of natural interference towards the wireless signal due to temperature and relative humidity (RH) factor [22]. From [23] and [24], we had found out that these two factors has affect the intensity level of our Wi-Fi signals in both $2.4 \mathrm{GHz}$ and $5 \mathrm{GHz}$. By referring to meteorological data provided by [25], this effect is very obvious especially during hot summer time in Okayama University due to higher RH percentage. During the throughput test, we had measured these values with RH meter and found out 
that higher RH value will degrade the performance of Wi-Fi throughput. Table 1 shows a comparison between different $\mathrm{RH}$ values during one of our tests. Further research regarding this interference is required to prove its effectiveness towards Wi-Fi signal.

\section{Table 1. Thermal Fade Effects Towards Wi-Fi Throughput}

\begin{tabular}{|c|c|c|c|}
\hline Test & Temperature & RH & Throughput \\
\hline 1 & $29^{\circ} \mathrm{C}$ & $56 \%$ & $60 \mathrm{Mbps}$ \\
\hline 2 & $29^{\circ} \mathrm{C}$ & $42 \%$ & $126 \mathrm{Mbps}$ \\
\hline
\end{tabular}

\section{Measurement Results}

In this section, we present throughput measurement results and discussions on them as follows:

- Comparison between indoor and outdoor

- Comparison between one-hop and two-hop

- Effects of walls

- Effect of 11 ac repeater

- Lectures Hall with Different AP Locations

- Effects of link distance

\subsection{Comparison between Indoor and Outdoor}

First, we compare throughput results between indoor and outdoor sites. Figure 20 and Figure 21 show the results for 11 ac using Buffalo WZR-G1750DHP and those for $11 \mathrm{n}$ using Buffalo WZR-G302H. They indicate that for $11 \mathrm{ac}$, indoor sites provide higher throughputs than outdoor sites and throughputs are gradually decreased as the longer distance. On the other hand, for $11 \mathrm{n}$, outdoor sites provide higher throughputs than indoor sites until $20 \mathrm{~m}$ distance. Throughputs by $11 \mathrm{ac}$ is about eight times by $11 \mathrm{n}$.

\subsection{Comparison between one-hop and two-hop}

Secondly, we compare throughput results between one-hop and two-hop communications. Figure 22 and Figure 23 show the results for 11ac using Buffalo WZRG1750DHP and those for 11n using Buffalo WZR-G302H. They indicate that for both protocols, throughputs of two-hop communications become about half of one-hop communications.

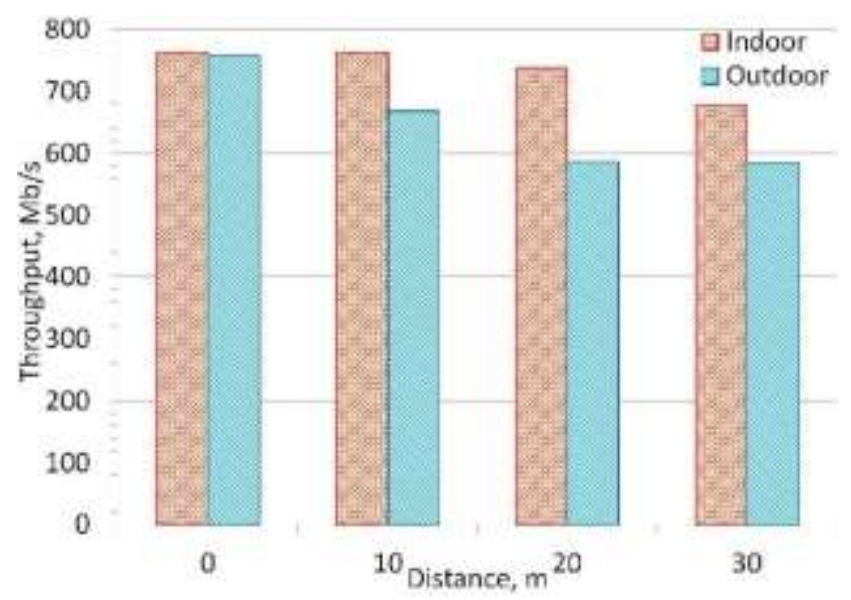

Figure 20. 11ac Throughput Comparisons between Indoor and Outdoor using Buffalo WZR-G1750DHP 


\subsection{Effects of Walls}

Thirdly, we evaluate throughput changes by wall obstacles along the link path. Figure 24 and Figure 25 show throughput changes when the number of walls increases from zero to two for $11 \mathrm{ac}$ and $11 \mathrm{n}$. For $11 \mathrm{ac}$, the throughput becomes half for one wall and one quarter for two walls from the one for no wall. While for $11 \mathrm{n}$, the drop rate is larger than that for $11 \mathrm{ac}$. We consider the reason comes from the difference on the number of MIMO antennas between both protocols.

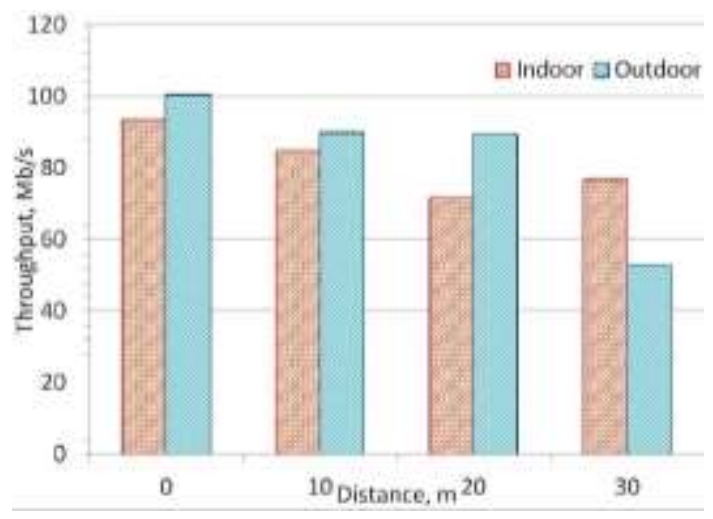

Figure 21. 11n Throughput Comparisons between Indoor and Outdoor using Buffalo WZR-G302H

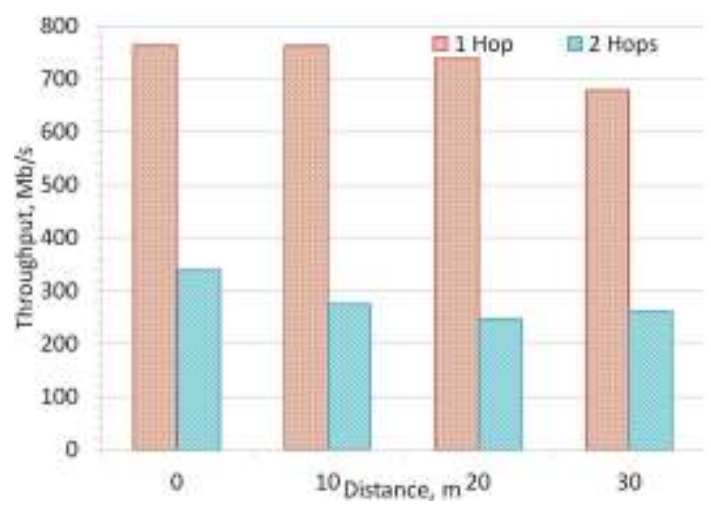

Figure 22. 11ac Throughput Comparisons between one-hop and two-hop using Buffalo WZR-G1750DHP

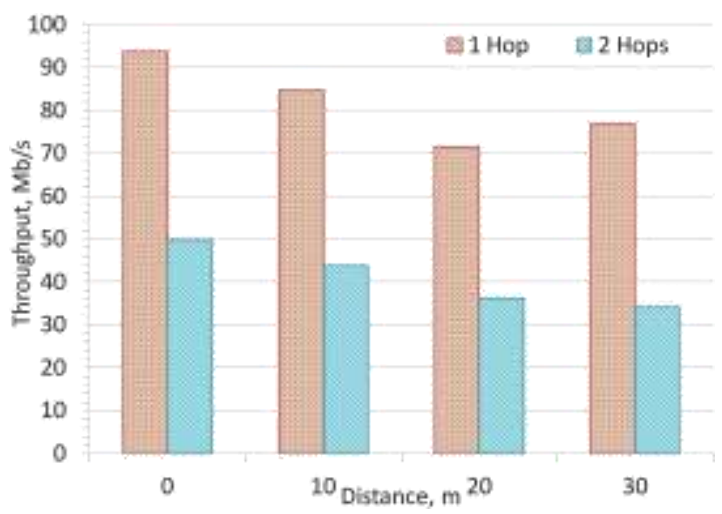

Figure 23. 11n Throughput Comparisons between one-hop and two-hop using Buffalo WZR-HP-G302H 
Then, we evaluate the throughput increase by using a repeater between the end nodes. Figure 26 compares the throughput with or without a repeater for the topology in Figure 3 and Figure 4. The results show that using the repeater, the throughput by WZR-G1750DHP 11 ac has increased by $7 \%$ and the throughput by WZR-HP-G302H $11 \mathrm{n}$ increased by $540 \%$. Thus, the effect of the repeater is confirmed.

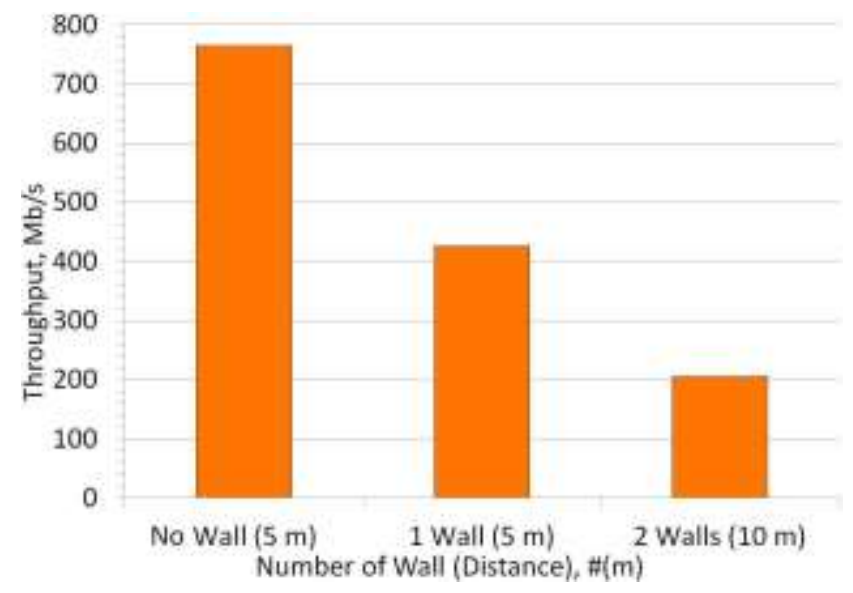

Figure 24. 11ac Throughput Change by Increasing \# of Walls in Indoor using Buffalo WZR-G1750DHP

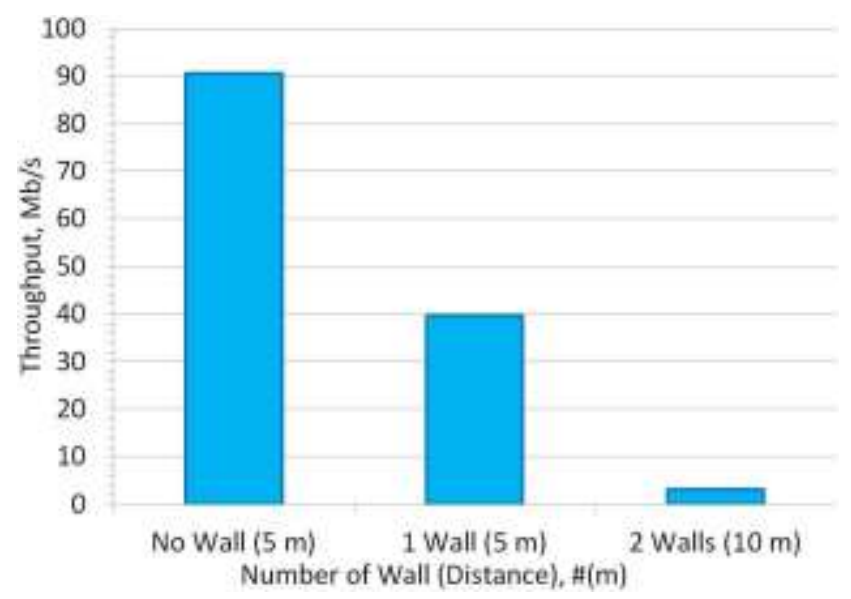

Figure 25. 11n Throughput Change by Increasing \# of Walls in Indoor using Buffalo WZR-HP-G302H

Lastly, we did the variable obstacles test with concrete walls using devices from Buffalo, I-O Data and NEC to evaluate the effects of wall obstacles towards $11 \mathrm{n}$ and $11 \mathrm{ac} \mathrm{Wi}-\mathrm{Fi}$ signal. The AP from I-O Data do not allow the repeater use for both 11ac and 11n. Figure 27 shows the effects of wall obstacle towards 11ac throughput from host to AP using different vendor. Vendor from Buffalo perform better than others under the test condition of no wall and one wall. For two walls test condition, NEC outperform other vendors at least $27 \%$. The maximum throughput for 11 ac USB Wi-Fi to AP at $8 \mathrm{~m}$ distance is about $291 \mathrm{Mbps}$. Under this no wall condition, this host has reach its top speed. The throughput of $11 \mathrm{ac}$ from host to AP can be summarized as Equation (2), with y represent the throughput and $\mathrm{x}$ represent the wall number from 0 to 2 .

$$
y=-64.695 x^{2}+57.085 x+258.28
$$


Figure 28 shows the effects of wall obstacle towards 11 ac throughput from AP to AP using different vendor. For this test, an average drop around $32 \%$ obtained per wall obstacle increased. The AP to AP's throughput is about double the host to AP performance.

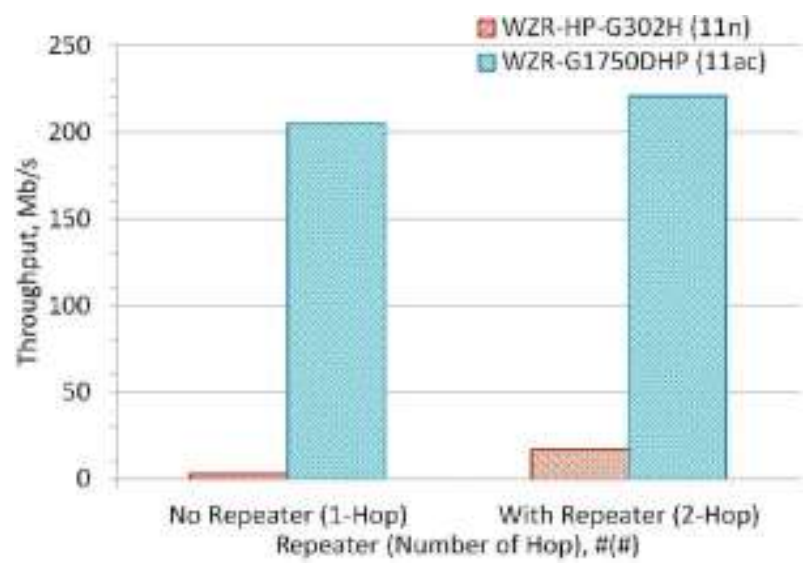

Figure 26. Effect of Repeater in Indoor with Two Walls using Buffalo WZRHP-G302H \& WZR-G1750DHP

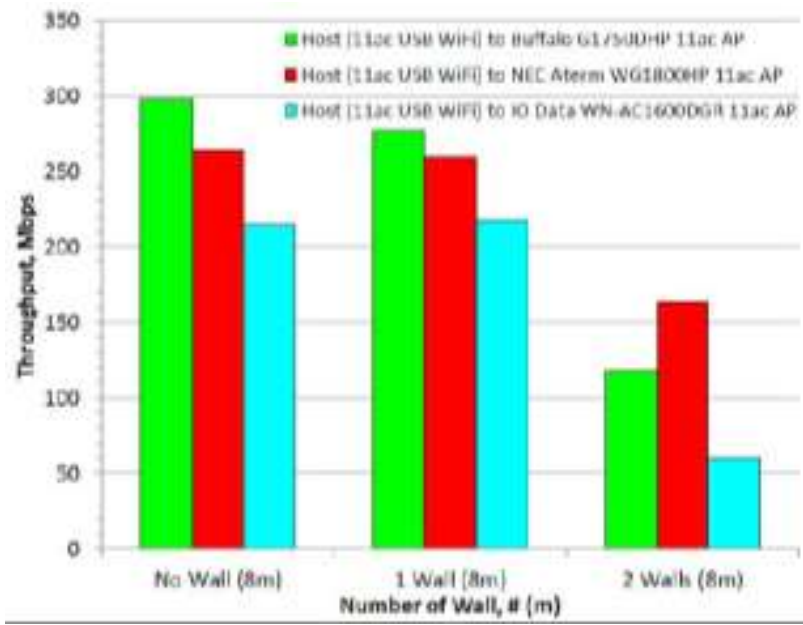

Figure 27. Effects of Wall Obstacle Towards 11ac Throughput from Host to AP using Different Vendor

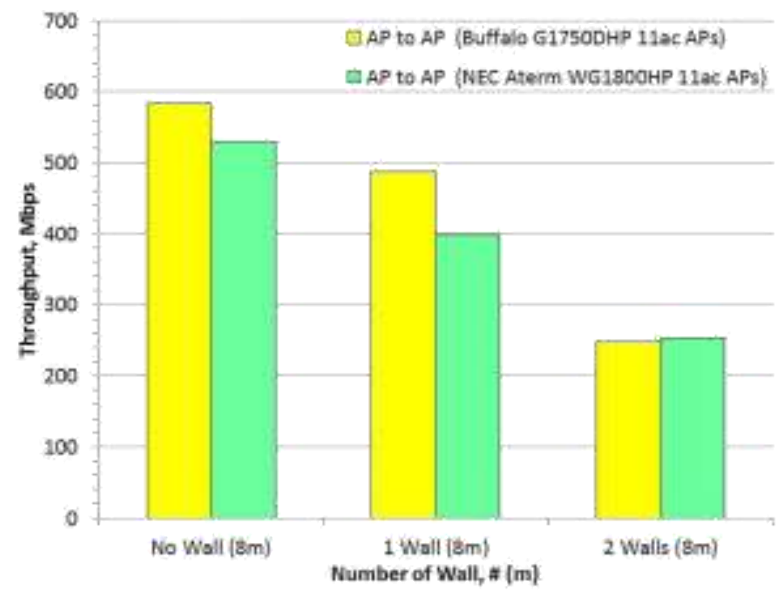

Figure 28. Effects of Wall Obstacle Towards 11ac Throughput from AP to AP using Different Vendor 
The throughput of $11 \mathrm{ac}$ from AP to AP can be summarized as Equation (3), with $y$ represent the throughput and $x$ represent the wall number from 0 to 2 .

$$
y=-39.205 x^{2}-74.465 x+555.92
$$

Figure 29 shows the effects of wall obstacle towards $11 \mathrm{n}$ throughput from host to AP using different vendor. I-O Data AP provide the best throughput under no wall and one wall conditions. Even though both the Buffalo G1750DHP and NEC WG1800HP AP are newer and better than the older Buffalo G302, they still having similar throughput results under all condition, this is due to the host has reach its maximum speed. The throughput are fully dependent on the build-in Wi-Fi model at the host side. The throughput of 11n host to AP can be summarized as Equation (4), with $y$ represent the throughput and $x$ represent the wall number from 0 to 2 .

$$
y=-2.78 x^{2}-14.46 x+102.97
$$

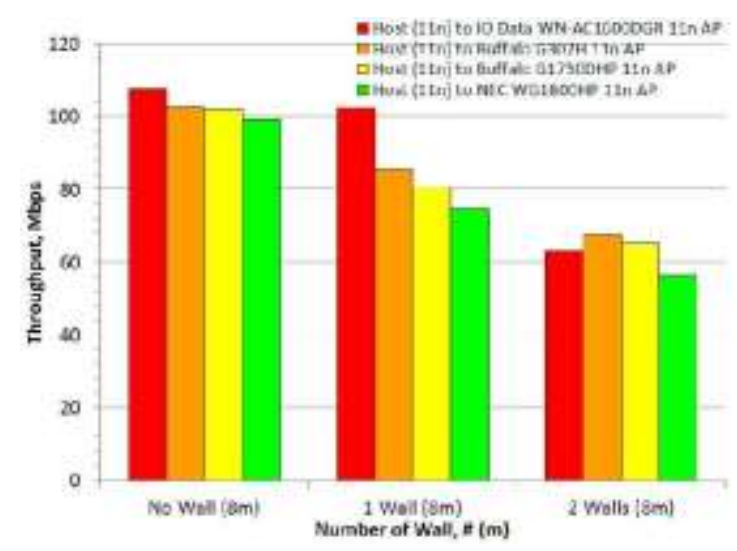

Figure 29. Effects of Wall Obstacle Towards 11n Throughput from Host to AP using Different Vendor

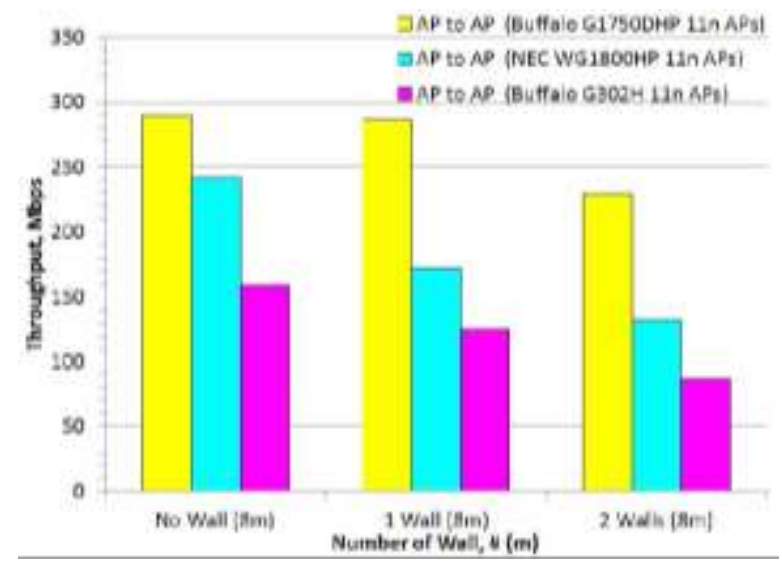

\section{Figure 30. Effects of Wall Obstacle Towards 11n Throughput from AP to AP using Different Vendor}

Figure 30 shows the effects of wall obstacle towards $11 \mathrm{n}$ throughput from AP to AP using different vendor. The newer Buffalo G1750DHP AP having the best throughput among 3 models of APs. It can provide the similar throughput under both no wall and one wall condition. Both NEC and Buffalo G302H AP having linear throughput results as numbers of wall increased. The older Buffalo G302H 11n AP having the lowers throughput due to its older hardware technology. The throughput of 11n AP to AP can be summarized 
as Equation (5), with $y$ represent the throughput and $x$ represent the wall number from 0 to 2 .

$$
y=-4.775 x^{2}-30.775 x+229.99
$$

\subsection{Effect of 11ac Repeater}

Fourthly, we evaluate throughput improvements by adopting an $11 \mathrm{ac}$ repeater between the server and two clients when a wall obstacle separates them as in Figure 5 and Figure 6. The devices from the three vendors are used, where those from I-O Data do not allow the repeater use (only one hop communications). For $11 \mathrm{n}$ devices at clients, both of the external USB Wi-Fi device and the build-in device in the client PC are evaluated. Table 2 shows the throughput measurement results. The results indicate:

a. NEC devices provide the highest throughput for one-hop communications, and for twohop communications using the external USB Wi-Fi device.

b. Buffalo devices provide the highest throughput when build-in devices in PCs are used with the 11 ac repeater, which can be the most conventional.

c. The adoption of an $11 \mathrm{ac}$ repeater improves the throughput for any case.

Table 2. Effect of 11ac Repeater in Indoor with One wall using Devices from Three Vendors

\begin{tabular}{|l|c|c|c|c|}
\hline \multirow{2}{*}{ Vendor } & \multicolumn{4}{|c|}{ Throughput (Mbps) } \\
\cline { 2 - 5 } & USB Wi-Fi & \multicolumn{2}{c|}{ Build-in Wi-Fi } \\
\hline Repeater & No & Yes & No & Yes \\
\hline Buffalo & 107.28 & 128.6 & 73.37 & 97.25 \\
\hline I-O Data & 138.53 & - & 79.47 & - \\
\hline NEC & 194.83 & 199.8 & 85.42 & 93.33 \\
\hline
\end{tabular}

\subsection{Lectures Hall with Different AP Locations}

Fifthly, we use 11ac USB Wi-Fi devices and build-in 11n Wi-Fi devices in the notebook PCs to compare throughputs for the different number of APs, different number of hosts as well as different locations in a big lecture room in our campus.

\subsubsection{Ideal Case}

In this case, Figure 31 shows the throughput results for 11ac. Two APs using different channels can double the throughput of one AP.

\subsubsection{Practical Case}

For this case, Figure 32 shows the throughput results when the number of 11ac's APs is changed from one to three. The throughput increases with the number of APs. However, for two-AP cases, the throughput decreases tremendously when seven or more hosts join.

The reason may come from the Wi-Fi interference increase, whose analysis will be in our future works. 


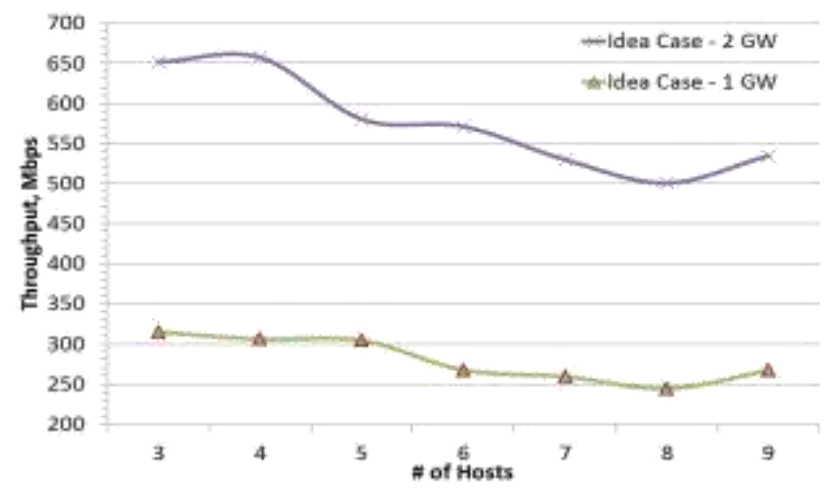

Figure 31. 11ac Throughput Comparison between One AP and Two APs for Ideal AP Location

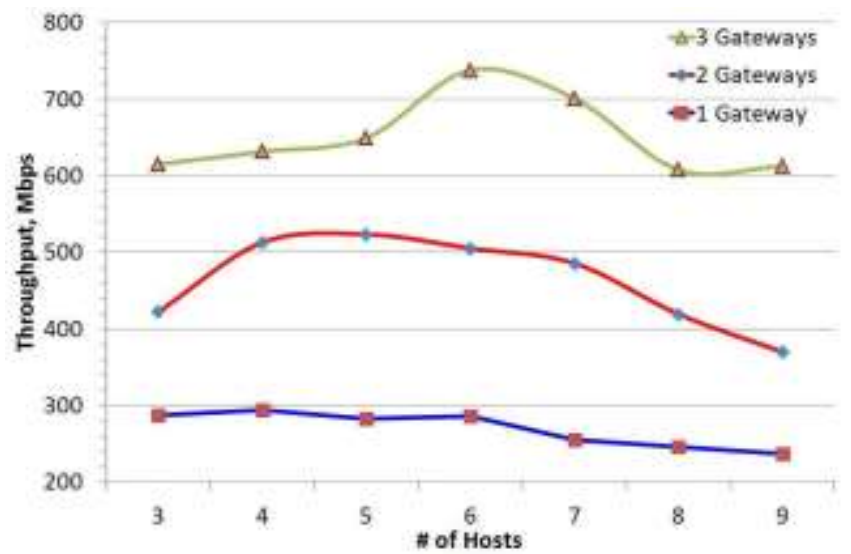

Figure 32. 11ac Throughputs with One to Three APs for Practical AP Location

Figure 33 shows the comparison of the 11ac throughputs between ideal and practical AP locations. For 1 gateway throughput, ideal case is better than practical case for 3 to 5 hosts position and they make the different between both ideal and practical cases. After 5 hosts, AP position does not make better performance. For 2 gateways throughput, ideal case is better than practical case due to shorter distance between hosts and gateways. For 9 hosts ideal case in both 1 and 2 gateways, the throughput suddenly goes up due to host number 9 is nearer to its associated AP. In overall, they show that throughputs are greatly affected by AP locations for two-AP cases and are not affected for one-AP cases.

Figure 34 shows the $11 \mathrm{n} 5 \mathrm{GHz}$ throughputs result when the number of $11 \mathrm{n}$ 's APs is changed from one to three. The throughput increases with the number of APs. Figure 35 shows the $11 \mathrm{n} 2.4 \mathrm{GHz}$ throughputs result when the number of 11n's APs is changed from one to three. The throughput decreases with the number of APs. For 2 gateways case, some interference occur during 7 to 8 hosts test, as a result, throughput drops a lot. 


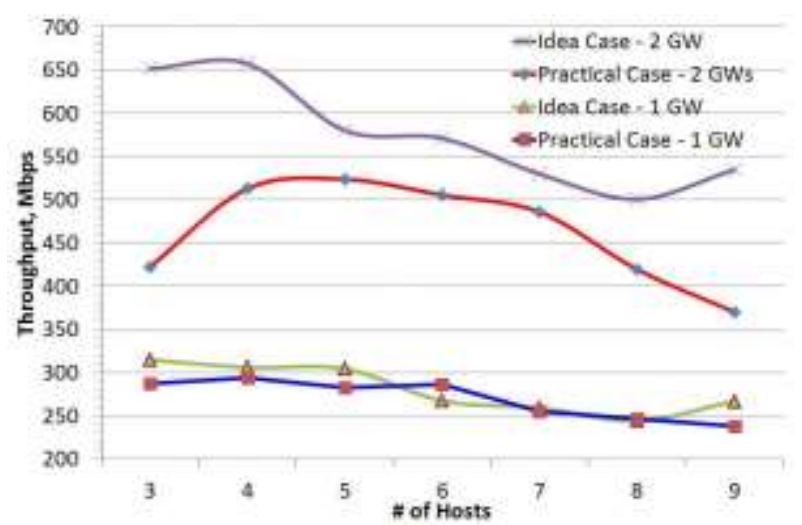

Figure 33. 11ac Throughputs Comparison between Ideal and Practical AP Locations

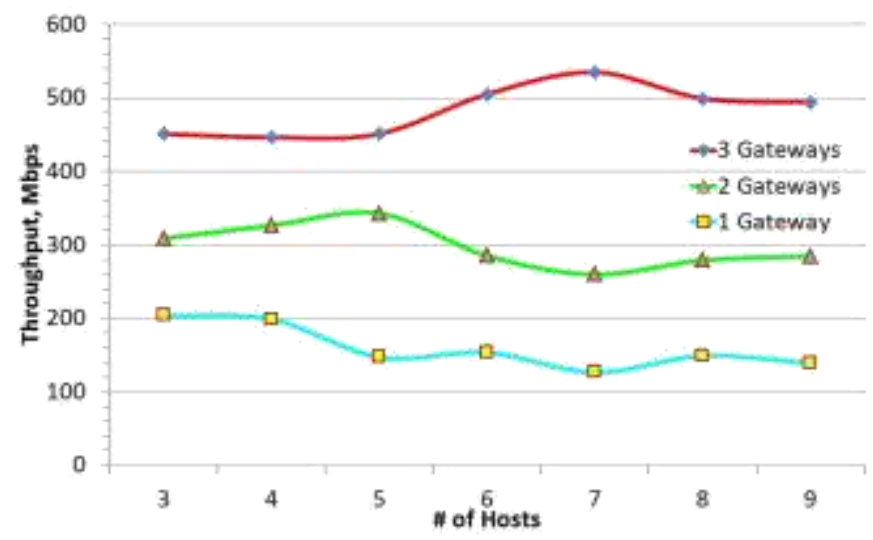

Figure 34. Effects of Variable Gateways Towards 11n 5GHz Throughput in Practical Case

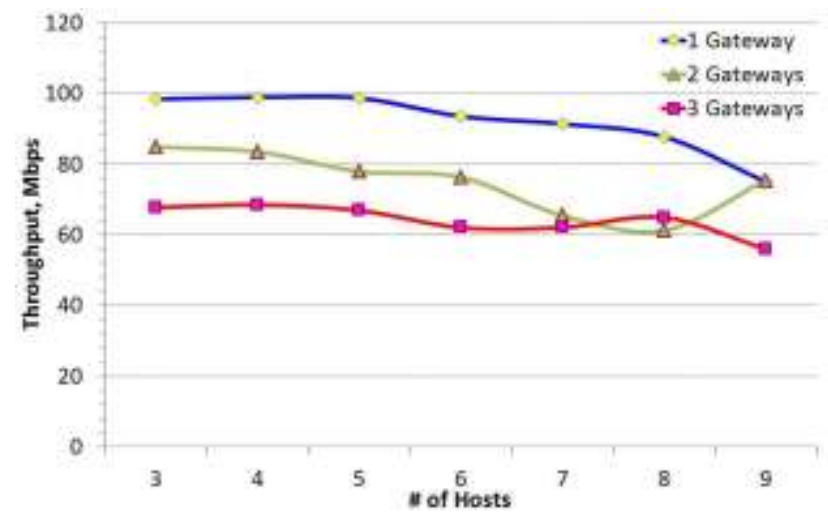

Figure 35. Effects of Variable Gateways towards 11n 2.4GHz Throughput in Practical Case

\subsection{Effects of Link Distance}

Finally, we evaluate throughput changes when the link distance increases in one-hop communications.

\subsubsection{Comparison Between 11ac and 11n}

Figure 36 shows the results for $11 \mathrm{ac}$ and $11 \mathrm{n}$ when the link distance increases from $0 \mathrm{~m}$ to $100 \mathrm{~m}$ in outdoor sites. The throughput for $11 \mathrm{ac}$ decreases from $758 \mathrm{Mbps}$ at $0 \mathrm{~m}$ to 
$182 \mathrm{Mbps}$ at $100 \mathrm{~m}$, and that for $11 \mathrm{n}$ decreases from $100 \mathrm{Mbps}$ at $0 \mathrm{~m}$ to $12 \mathrm{Mbps}$ at $100 \mathrm{~m}$. This means 11ac provides about eight times faster throughput of $11 \mathrm{n}$.

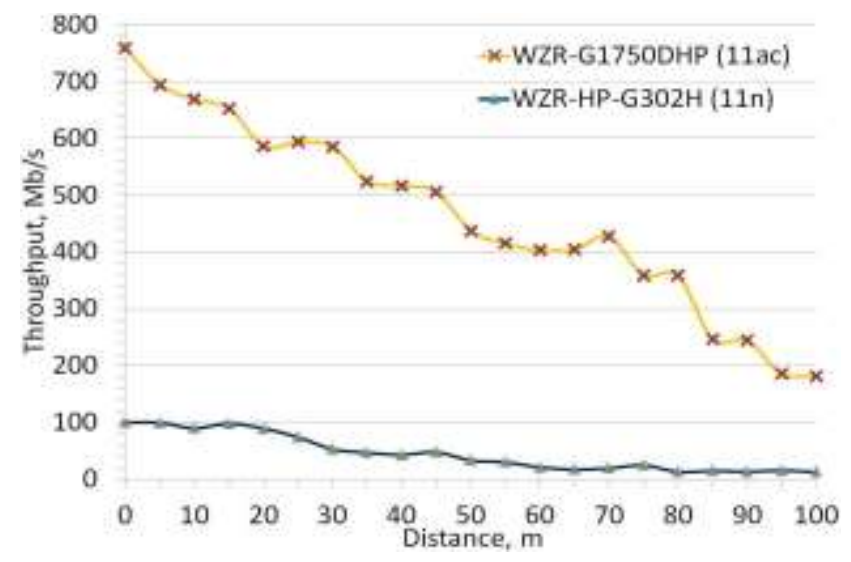

Figure 36. 11ac Throughput Change by Increasing Link Distance in Outdoor using Buffalo WZR-G1750DHP\& WZR-HP-G302H

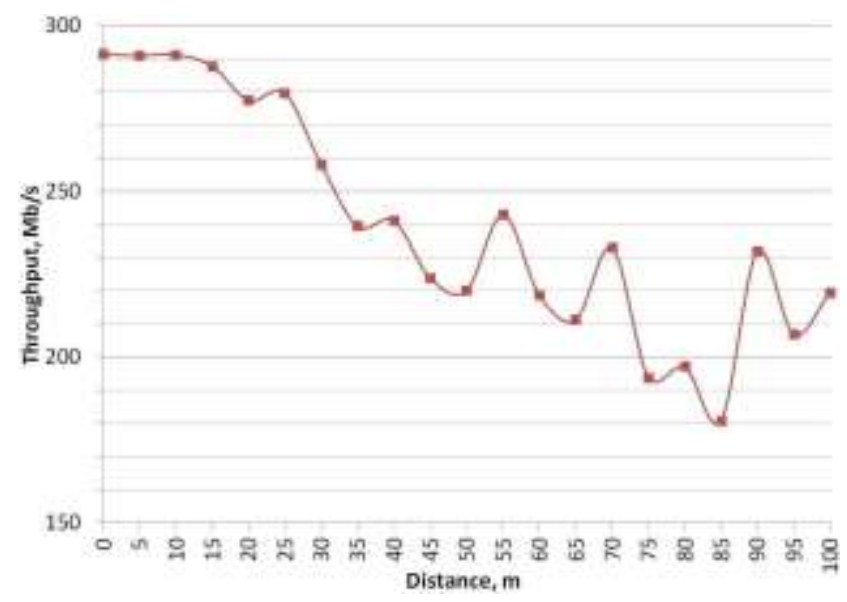

Figure 37. 11ac Throughput Change by Increasing Link Distance in Outdoor using Buffalo WZR-G1750DHP \& WI-U2-866D USB Wi-Fi

We had generated an 11ac AP throughput link distance equation as in Equation (6), with $y$ represent the throughput and $x$ represent the distance from 0 to $100 \mathrm{~m}$.

$$
y=-0.0266 x^{2}-31.5812 x^{1 / 2}+762.4162
$$

\subsubsection{Comparison between Different Vendors}

In this part, we compared throughputs between different wireless device vendors when the link distance increases in outdoor sites. In each experiment, the both $11 \mathrm{ac}$ USB Wi-Fi device and AP from the same vendors used for each test. Figure 37, Figure 38, and Figure 39 show throughput changes using devices from Buffalo, I-O Data and NEC respectively. Here, we note that the results are affected by interferences by others nearby Wi-Fi signals sharing the similar frequency spectrum at certain distances. The results by Buffalo and I-O Data similarly decrease as the link distance increases, while the results by NEC are more stable for distance changes. From these vendors, we had generated a 11 ac USB Wi-Fi throughput link distance equation as in Equation (7), with $y$ represent the throughput and $x$ represent the distance from 0 to $100 \mathrm{~m}$.

$$
y=-0.0097 x^{2}-0.7014 x^{1 / 2}+293.1530
$$




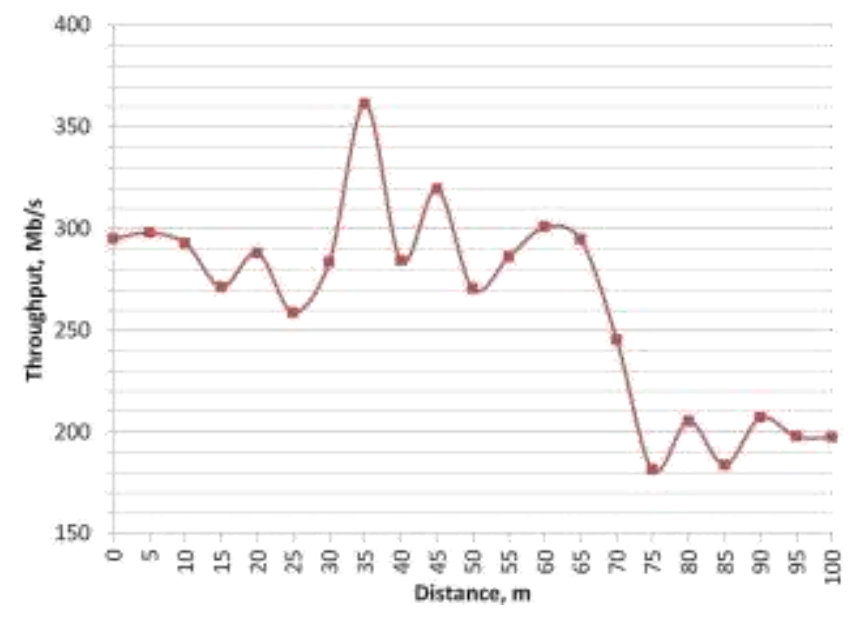

Figure 38. 11ac Throughput Change by Increasing Link Distance in Outdoor using IO Data WN-AC1600DGR \& WN-AC867U USB Wi-Fi

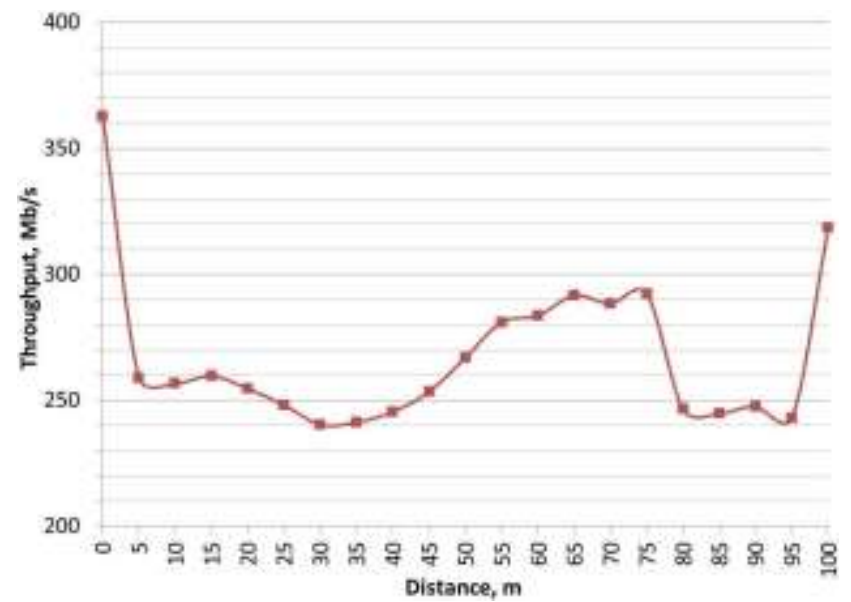

Figure 39. 11ac throughput Change by Increasing Link Distance in Outdoor using NEC WG1800HP \& WL900U USB Wi-Fi

\section{Conclusion and Future Works}

This paper has presented TCP throughput measurement results using IEEE 802.11ac/11n devices from three vendors implementing IEEE $802.11 \mathrm{ac} / 11 \mathrm{n}$ protocols in various conditions including different TCP parameters, one or two-hop communications, wall obstacle existences, different link distances, the use of a repeater, and big room with different AP locations. They show that TCP throughputs are affected by vendors, communication conditions, and AP's location. In future works, we will continue throughput measurements in different conditions, and present a computational model to describe these features for precise wireless network simulations.

\section{References}

[1] M. S. Gast, 802.11ac: a survival guide, 1st ed., O’Reilly, (2013).

[2] C. Choon Chew, N. Funabiki, T. Nakanishi and K. Watanabe, "Throughput Measurements Using IEEE802.11n and 11ac Devices for High-Speed Wireless Networks", The 2013 (64th) Chugoku-branch Joint Convention of Institutes of Electrical and Information Engineers, (2013) October, pp. 232-233.

[3] C. Choon Chew, N. Funabiki, T. Nakanishi and K. Watanabe, "Throughput Measurements Using IEEE 802.11ac Devices for High-Speed Wireless Networks", IEICE Technical Report, vol. 113 no. 463, CAS2013-94, (2014) March, pp. 23-28. 
[4] C. Choon Chew and N. Funabiki, "Throughput Measurements in Big Room with Different Access Point Locations for IEEE 802.11ac Wireless Networks", IEICE Society Conference 2014, (2014) September, pp. S-38-39.

[5] Buffalo Inc., Buffalo WZR-HP-G302H Product Manual, http://buffalo.jp/download/manual/w/wzr hpg302h.html/.

[6] Buffalo Inc, AirStation WZR-1750DHP/WZR-1166DHP User Manual, http://manual.buffalo. jp/bufdoc/35013154-02 EN.pdf/.

[7] I-O Data Device Inc., IO Data Air Port WN-AC1600DGR Manual, http://www.iodata.jp/lib/ manual/wnac1600dgr/index.htm/.

[8] NEC Access Technica Ltd., Aterm Function Guide, http://www.aterm.jp/function/wg1800hp/ index.html/.

[9] NEC Access Technica Ltd., Aterm WG1800HP/ WG1400HP Operation Manual, http://www. aterm.jp/function/wg1800hp/index.html/.

[10] Buffalo Inc., AirStation WI-U2-866D User Manual, http://manual.buffalo.jp/buf-doc/350129 65-03 EN.pdf/.

[11] I-O Data Device Inc., IO Data WN-AC867U Manual, http://www.iodata.jp/lib/manual/wn-acu/ index.htm/.

[12] NEC Access Technica Ltd., NEC Aterm WL900U Manual, http://www.aterm.jp/support/manual/ wl900u/index.html.

[13] Wikipedia, Laptop - Heating and Cooling, http:/ /en.wikipedia.org/wiki/Laptop\#Heating and cooling/

[14] Buffalo Inc., AirStation WI-U2-866D User Manual, http://manual.buffalo.jp/buf-doc/350129 65-03 EN.pdf, pp. 55.

[15] Microsoft, View and Connect to Available Wireless Networks, http://windows.microsoft. com/enus/windows/view-connect-available-wireless-networks\#1TC=windows-7.

[16] Speed Guide Inc., SG TCP Optimizer, http:// www.speedguide.net/sg tools.php.

[17] A. S. Tanenbaum and D. J. Wetherall, "Computer Networks", 5th ed., Prentice Hall, (2011).

[18] ACD.net, Iperf Speed Testing, http://support. acd.net/wiki/index.php?title=Iperf Speed Testing/.

[19] Speed Guide Inc., SG RWIN/BDP Calculator, http://www.speedguide.net/bdp.php.

[20] Wi-Fi Channel Scanner, http:// www.wifichannelscanner.com/index.html/.

[21] Farproc, Wifi Analyzer v3.8.7, http://a.farproc. com/wifi-analyzer.

[22] Wikipedia, Thermal fade, http://en.wikipedia. org/wiki/Thermal fade.

[23] N. Yackerson, "Influence of air humidity on microwave depolarisation in semi-arid areas", Microwaves, Antennas and Propagation, IEE Proceedings, vol. 145, no. 2, (1998) April, pp. 147-152.

[24] Navy-Marine Corps Military Auxiliary Radio System Operator, "Navy Electricity and Electronics Training Series Modules 6 - Radio Wave Propagation," http://www.navymars.org/ national/training/nmo courses/NMO1/module10/ 14182ch2.pdf, pp. 2-33-2-37.

[25] Japan Meteorological Agency, Past weather data search, http://www.data.jma.go.jp/obd/stats/etrn/index.php. 